\title{
Recombinant Haemonchus contortus 24 kDa excretory/ secretory protein (rHcES-24) modulate the immune functions of goat PBMCs in vitro
}

\author{
Javaid Ali Gadahi ${ }^{1,2, *}$, Baojie $\mathrm{Li}^{1}$, Muhammad Ehsan ${ }^{1, *}$, Shuai Wang ${ }^{1}$, Zhenchao \\ Zhang ${ }^{1}$, Yujian Wang ${ }^{1}$, Muhammad Waqqas Hasan ${ }^{1}$, Ruofeng Yan ${ }^{1}$, Xiaokai Song ${ }^{1}$, \\ Lixin $\mathbf{X u}^{1}$ and Xiangrui $\mathbf{L i}^{1}$ \\ ${ }^{1}$ College of Veterinary Medicine, Nanjing Agricultural University, Nanjing, PR China \\ 2 Department of Veterinary Parasitology, Sindh Agriculture University Tandojam, Pakistan \\ * These authors have contributed equally to this work \\ Correspondence to: Xiangrui Li, email: lixiangrui@njau.edu.cn
}

Keywords: H. contortus, ESP, 24 kDa, cytokines, proliferation, cell migration, Immunology and Microbiology Section, Immune response, Immunity

Received: September 30,2016 Accepted: November 15,2016 Published: November 21, 2016

\section{ABSTRACT}

A 24 kDa protein is one of the important components in Haemonchus contortus (barber pole worm) excretory/secretory products (HcESPs), which was shown to have important antigenic function. However, little is known about the immunomodulatory effects of this protein on host cell. In the present study gene encoding $24 \mathrm{kDa}$ excretory/secretory protein (HcES-24) was cloned. The recombinant protein of HCES-24 (rHcES-24) was expressed in a histidine-tagged fusion protein soluble form in Escherichia coli. Binding activity of rHcES-24 to goat PBMCs was confirmed by immunofluorescence assay (IFA) and its immunomudulatory effect on cytokine secretion, cell proliferation, cell migration and nitric oxide production were observed by co-incubation of rHcES-24. IFA results revealed that rHcES-24 could bind to the PBMCs. The interaction of rHcES-24 increased the production of IL4, IL10, IL17 and cell migration in dose dependent manner. However, rHcES-24 treatment significantly suppressed the production of IFNy, proliferation of the PBMC and Nitric oxide (NO) production. Our findings showed that the rHcES-24 played important regulatory effects on the goat PBMCs.

\section{INTRODUCTION}

Haemonchosis is a disease of the small ruminant caused by a nematode parasite Haemonchus contortus ( $H$. contortus); it is most important and alarming challenges to the small ruminant's production. The infection of the $H$. contortus could cause high economic losses worldwide [1]. H. contortus is a blood feeding parasite and penetrates into the abomasal mucosa to feed the blood of the host and causing the anemia and decreased total plasma protein $[2$, $3]$.

H. contortus excretory/secretory (ES) molecules probably play important roles in the host-parasite interaction during infection process [4]. Previous studies revealed abundant expression of these molecules including sperm-coating protein (SCP)-like protein in the bloodfeeding stages of $H$. contortus $[5,6]$. These protein are found in a wide range of organism included arthropods, nematodes, flukes and plants [7-11] and considered prominent proteins in ES products [4]. In parasitic nematodes, Ancylostoma-secreted proteins (ASPs) were first described and reported that these proteins were abundant in the ES products of the infective stage (L3) assumed that, these proteins play a vital role in transition from free living to parasitic stage of hook worm $[12,13]$.

In $H$. contortus, two SCP proteins HcES-24 and Hc-40 have been recognized from the ES products [1416]. HcES-24 was first identified as a low molecular weight antigen recognized by hyperimmune sera from the experimentally infected sheep with L3 and also same protein was identified from the ESPs of adult worm [17, 18]. In distinction to hookworms, ES-24 was identified in L4 and adult worms but not in eggs or L3s [15].

Previously we identified, that the $H$. contortus excretory and secretory products (HcESPs) displayed suppressive potential on the goat PBMCs in vitro. HcESPs 
inhibited the productions of IL-4, IFN- $\gamma$, increased the suppressive cytokine IL-10, enhanced the inflammatory modulator IL-17, suppressed the production of chemical factor NO, decreased the cell proliferation and activated the cell migration [19]. In our previous proteomic study of $H$. contortus excretory and secretory products (HcESPs) binding to goat PBMCs, SCP like protein was identified as a interacting protein to goat PBMCs at L4 to adult stages of $H$. contortus in vivo [20]. Binding of this protein to goat PBMCs at multiple stages in vivo indicated its role in the immune modulation. In the current study, the gene encoding $24 \mathrm{kDA}$ (HcES-24) was cloned and the recombinant protein of HcES-24 (rHcES-24) was used to evaluate its regulatory effects on the goat PBMCs.

\section{RESULTS}

\section{Cloning of HcES-24 gene}

The amplicon of HcES-24 gene were successfully isolated by PCR of $H$. contortus cDNA with specific primers as designed above and a fragment of the correct size of $609 \mathrm{bp}$ was obtained. The recovered PCR product was purified and successfully cloned into pMD19-T cloning vector which was confirmed by restriction enzyme digestion with HindIII/EcoRI restriction site enzymes.

\section{Construction and identification of the recombinant pET-32a (+)-HcES-24}

The correct fragment of HcES-24 after sequencing was then inserted into HindIII/EcoRI sites of pET32a $(+)$ vector. The recombinant plasmid was confirmed with restricted digestion . The digestion of recombinant pET32a-HcES-24 produced a fragment of about 609 bp which is equal to molecular mass of HcES-24. These results indicated that HcES-24 has been successfully inserted into $\mathrm{pET} 32 \mathrm{a}$ vector.

\section{Sequence and phylogenetic analysis of HcES-24}

The isolated sequences were confirmed as HcES24 gene by BLASTx, ORF contains 609 bp and encodes 202 amino acids. Multiple sequence alignment and Phylogenetic tree of the deduced protein sequence of HcES-24 with available sequences on NCBI database is shown in Figure 1. The multiple alignments of the deduced amino acid sequence indicated that HcES-24 was most closely related to $H$. contortus SCP extracellular domain containing protein (98\%), H. contortus $24 \mathrm{kDa}$ excretory/ secretory protein $(97 \%), H$. contortus cap-2 (94\%), $H$. contortus cap-3 (93\%) and H. contortus cap-4 (78\%).

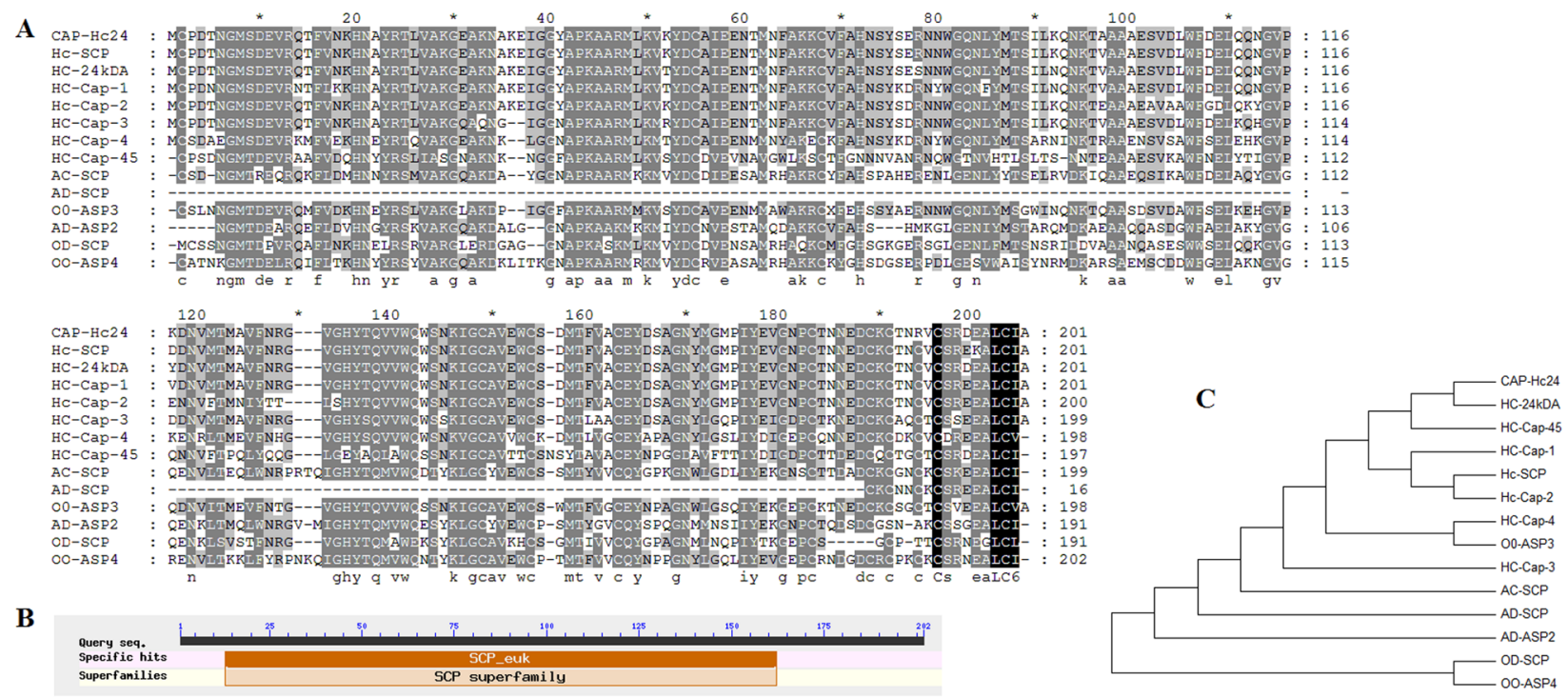

Figure 1: Multiple alignment of amino acid sequence of HcES-24 . A. The amino acid sequence of HcES-24 aligned with CAP genes reported in the NCBI database HC-SCP: H, contortus SCP extracellular domain containing protein CDJ92089.1 (98\%), HCES-24 kDA: H, contortus 24 kDa excretory/secretory protein AAC47714.1 (97\%), HC-Cap-1: H, contortus cap-1 ALA23451.1 (95\%), Hc-Cap-2: H, contortus cap-2 ALA23452.1 (94\%), Hc-Cap-3: H, contortus cap-3 ALA23454.1 (93\%), Hc-Cap-4: H, contortus cap-4 ALA23464.1 (78\%), Hc-Cap-45: H, contortus cap-45 ALA23426.1 (69\%), AC-SCP: Ancylostoma ceylanicum SCP-like protein EPB75408.1 (71\%), AD-SCP: Ancylostoma duodenale SCP-like protein KIH59107.1 (6\%), OO-ASP3: Ostertagia ostertagi C-type single domain activation associated secreted protein ASP3 CAO00416.1 (81\%), AD-ASP: Ancylostoma duodenale secreted protein ASP-2 AAP41951.1 (64\%), ODSCP: Oesophagostomum dentatum SCP-like protein KHJ79965.1 (65\%), OO-ASP4: Ostertagia ostertagi two-domain activation associated secreted protein ASP4 CAO00417.1 (67\%). B. Putative conserved domain C. Phylogenetic tree of deduced amino acid sequences of HcCAP and other nematodes. 


\section{Expression and purification of rHcES-24}

The rHcES-24 protein expressed in E. coli (BL21) cells as a double His 6 tagged fusion protein was purified. The expressed product was detected in SDS-PAGE after staining with coomassie brilliant blue (Figure 2). A protein band of rHcES-24 expressed product was about $42 \mathrm{kDa}$ instead of the calculated molecular mass of $\sim 24 \mathrm{kDa}$ due to extra pET-32a vector.

\section{Detection of rHcES-24 by western blotting}

Western blot indicated that the recombinant protein was recognized by Rat anti rHcES-24, whereas, no protein was recognized by the normal rat serum (Figure 3 ).

\section{Binding of rHcES-24 to goat PBMCs}

Binding of rHcES-24 to goat PBMCs was confirmed by immunofluorescence assay. Nuclei were stained with

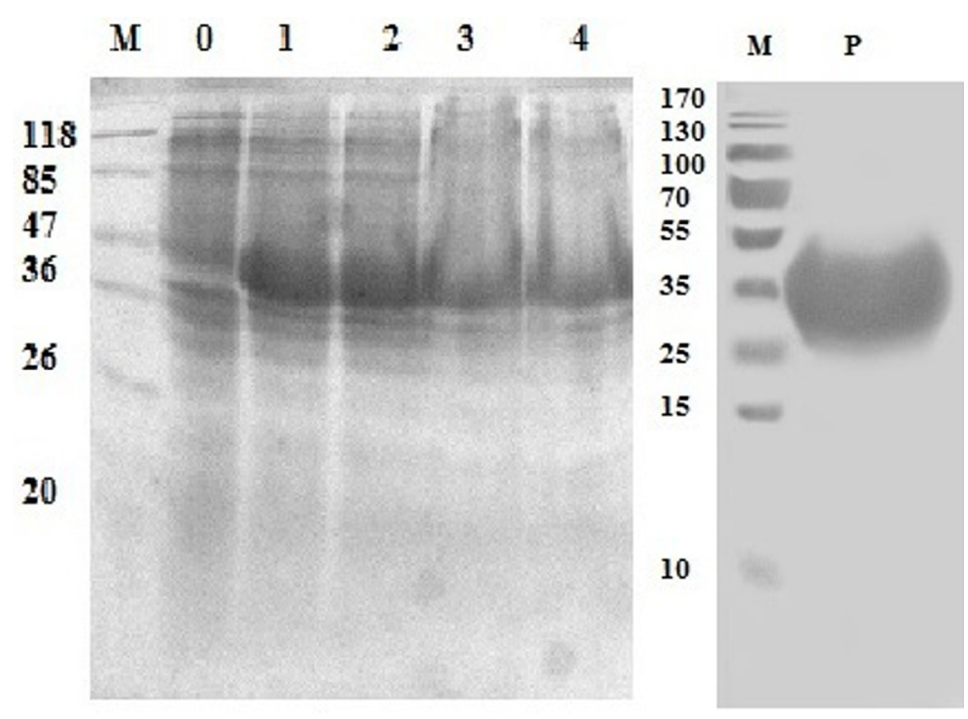

Figure 2: Expression and purification of rHcES-24 protein after induction with 1mM IPTG. Lane M: standard protein molecular weight marker, 0: recombinant expression vector before induction, Lane 1-4 expression after induction at different time point and Lane P: purified rHcES-24 protein.

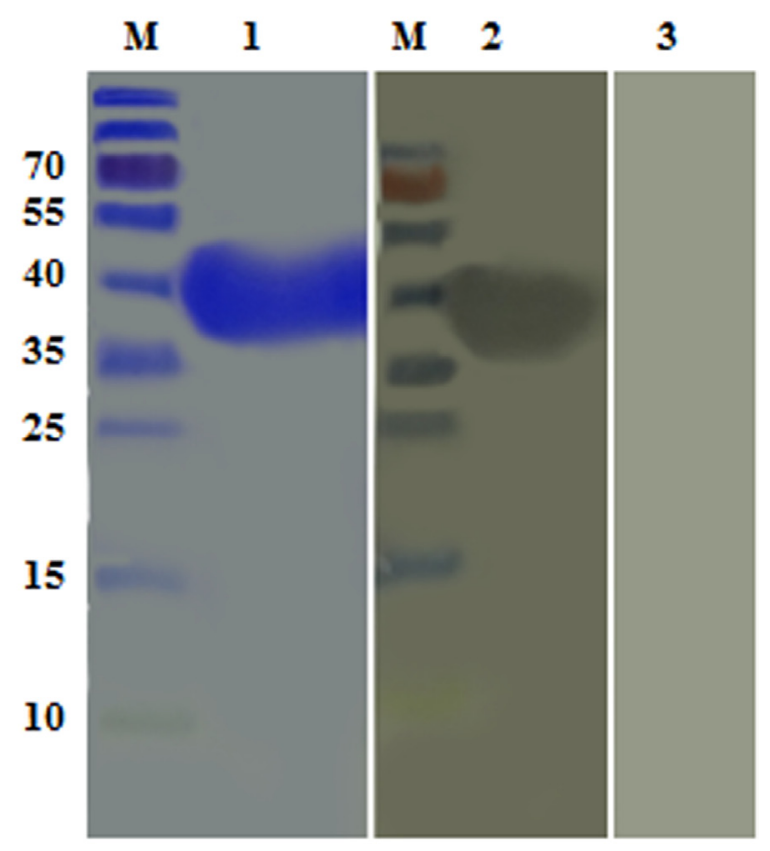

Figure 3: Western blot analysis of rHcES-24 . Purified rHcES-24 were electrophoresed in SDS-PAGE A. and then transferred to a membrane for western blot analysis with rat anti- rHcES-24 sera B. and normal rat sera $\mathbf{C}$. as control. 
DAPI (blue fluorescence), and confocal microscopy images revealed that the rHcES-24 was bound to the cell surface (red fluorescence). In the control group, no red fluorescence was observed (Figure 4).

\section{Detection of the cytokine levels by ELISA}

Effects of the rHcES-24 on the cytokine production was analyzed by ELISA and results showed that rHcES-24 was significantly modulated the cytokine secretion in dose dependent manner (Figure 5). Production of type 2 cytokine IL-4 was significantly increased in PBMCs incubated with 20 and $40 \mu \mathrm{g} / \mathrm{ml}$ of rHcES-24. No significant difference was observed between the control and PBMCs treated with rHcES-24 at the dose of 5 and $10 \mu \mathrm{g} / \mathrm{ml}$. In the present study, secretion of IL-10 and IL-17 were also increased by the rHcES-24 in dose dependent manner. On the contrary, type II interferon (IFN $\gamma$ ) was suppressed by rHcES-24 in dose dependent manner.

\section{Cell migration assay}

To evaluate the impact of rHcES-24 on cell migration, cell migration assay was conducted (Figure 6 ). The percentage of migrated cells was significantly increased in cell treated with different concentration of rHcES-24.

\section{Cell proliferation assay}

Cell counting kit (CCK8) was used to evaluate the effect of the rHcES-24 on the PBMC proliferation. rHcES-24 treatment significantly suppressed the proliferation of PBMC in dose dependent manner as compared with control group (Figure 7).

\section{Nitric oxide production assay}

Nitric oxide (NO) production by PBMCs treated with different concentration of rHcES-24 was measured by using the total nitric oxide assay kit. Results revealed that, rHcES-24 significantly suppressed the NO production by PBMCs (Figure 8).

\section{DISCUSSION}

The helminth ESPs contain profuse amounts of CAP-superfamily proteins commonly referred to as activation-associated secreted proteins (ASPs) or sperm coating protein (SCP). ASPs are considered to play a key role in the switching from the free-living to the parasitic stage [21]. Determination of three dimensional protein structures of helminth ASPs suggested a vital role in a different regulatory function including altering the immune response $[9,22,23]$. Previously two proteins

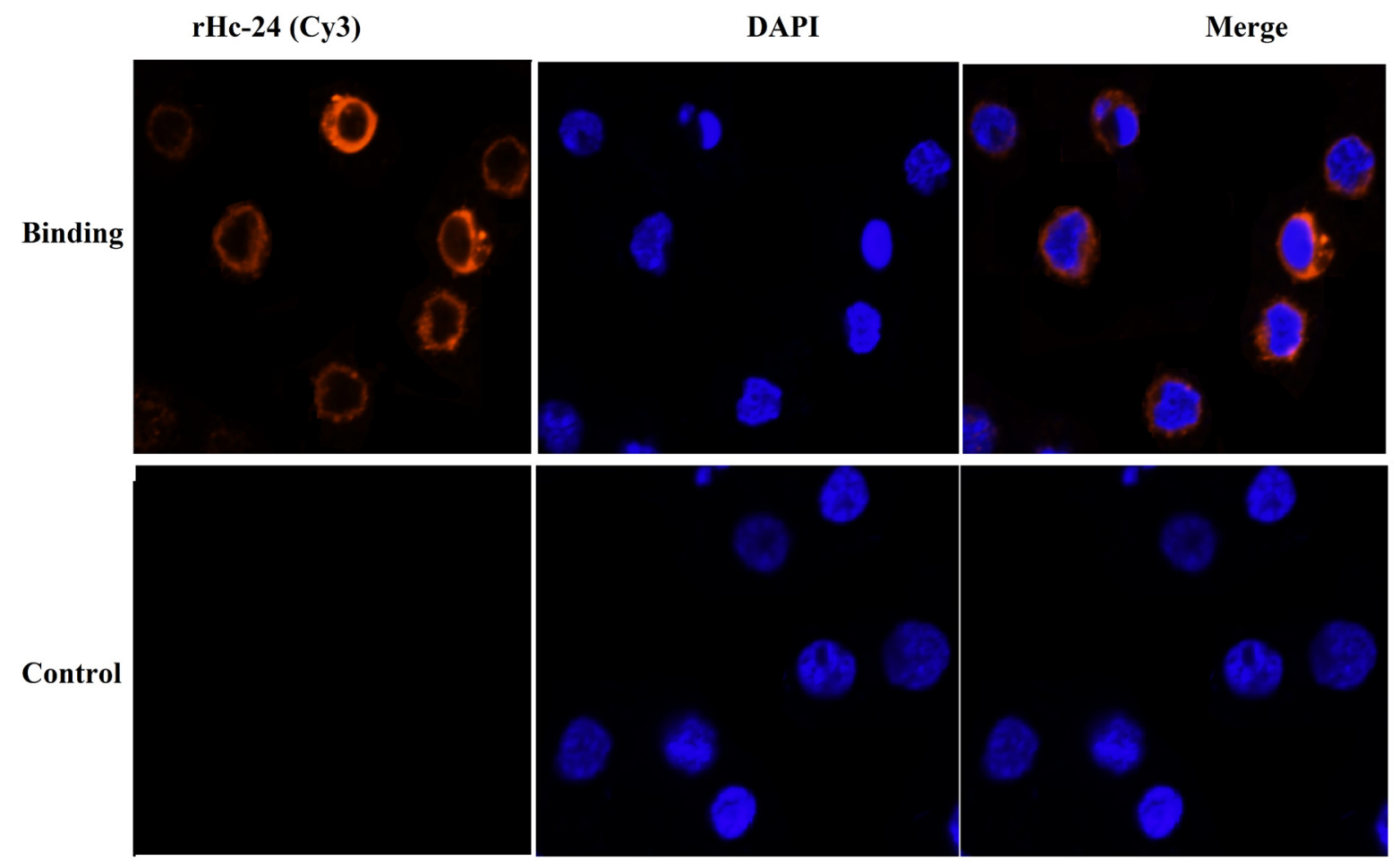

Figure 4: Binding of recombinant proteins rHcES-24 . The nuclei of the corresponding cells were visualized by DAPI (blue) staining. Staining of the target proteins (red) were visualized by Cy3-conjugated secondary antibody. Merge, overlap of red and blue channels. No red fluorescence was observed in control group. 

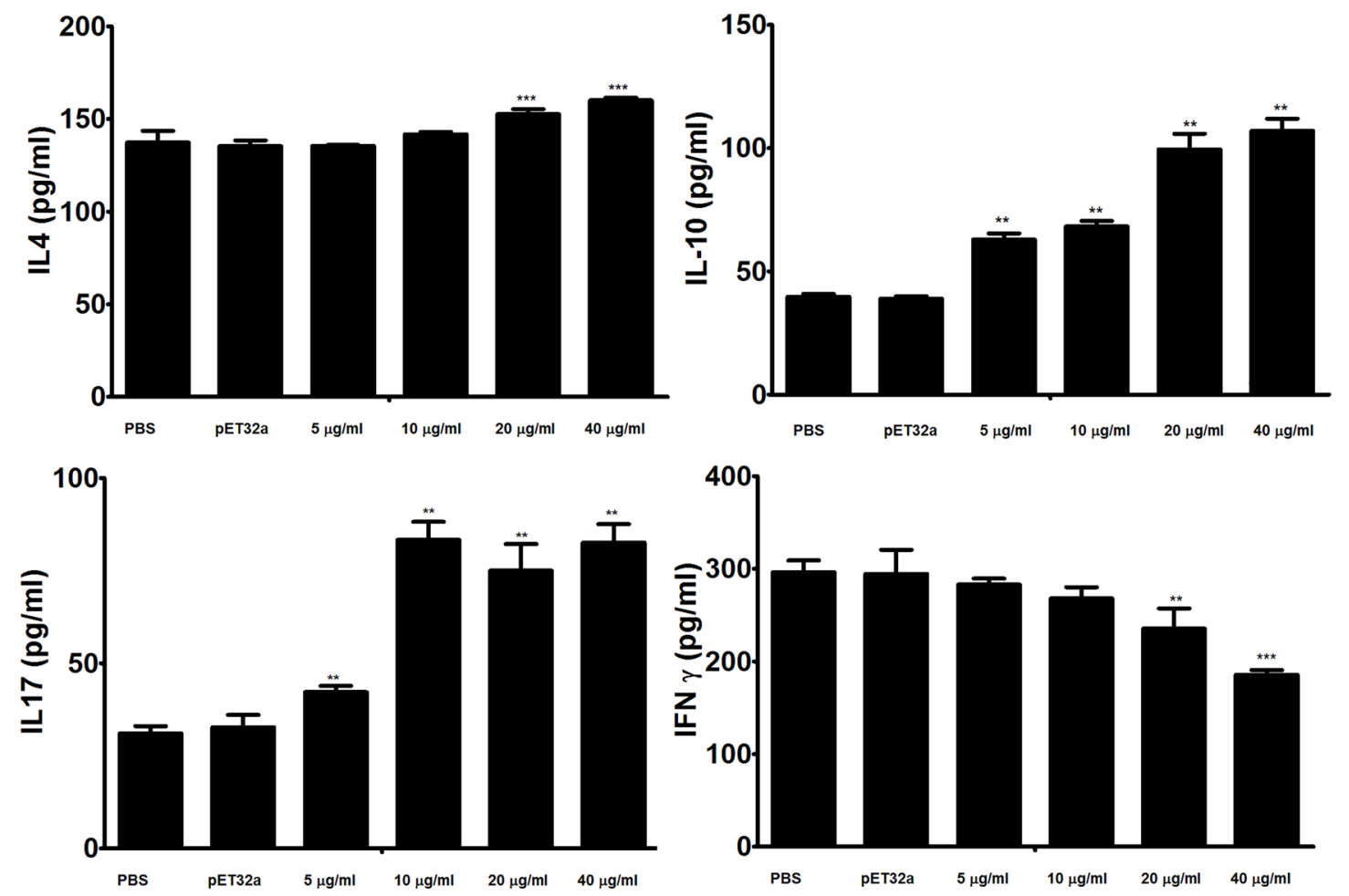

Figure 5: Analysis of the level of multiple cytokine production by PBMCs in vitro. PBMCs were stimulated with ConA (10 $\mu \mathrm{g} / \mathrm{ml}$ ) for $24 \mathrm{~h}$ in the presence or absence of various concentrations of rHcES-24 and pET32a. Cytokine secretion in the supernatant of cell cultures was quantified by ELISA. The data are representative of three independent experiments $(* * p<0.001$, and $* * * p<0.0001$ versus the control).

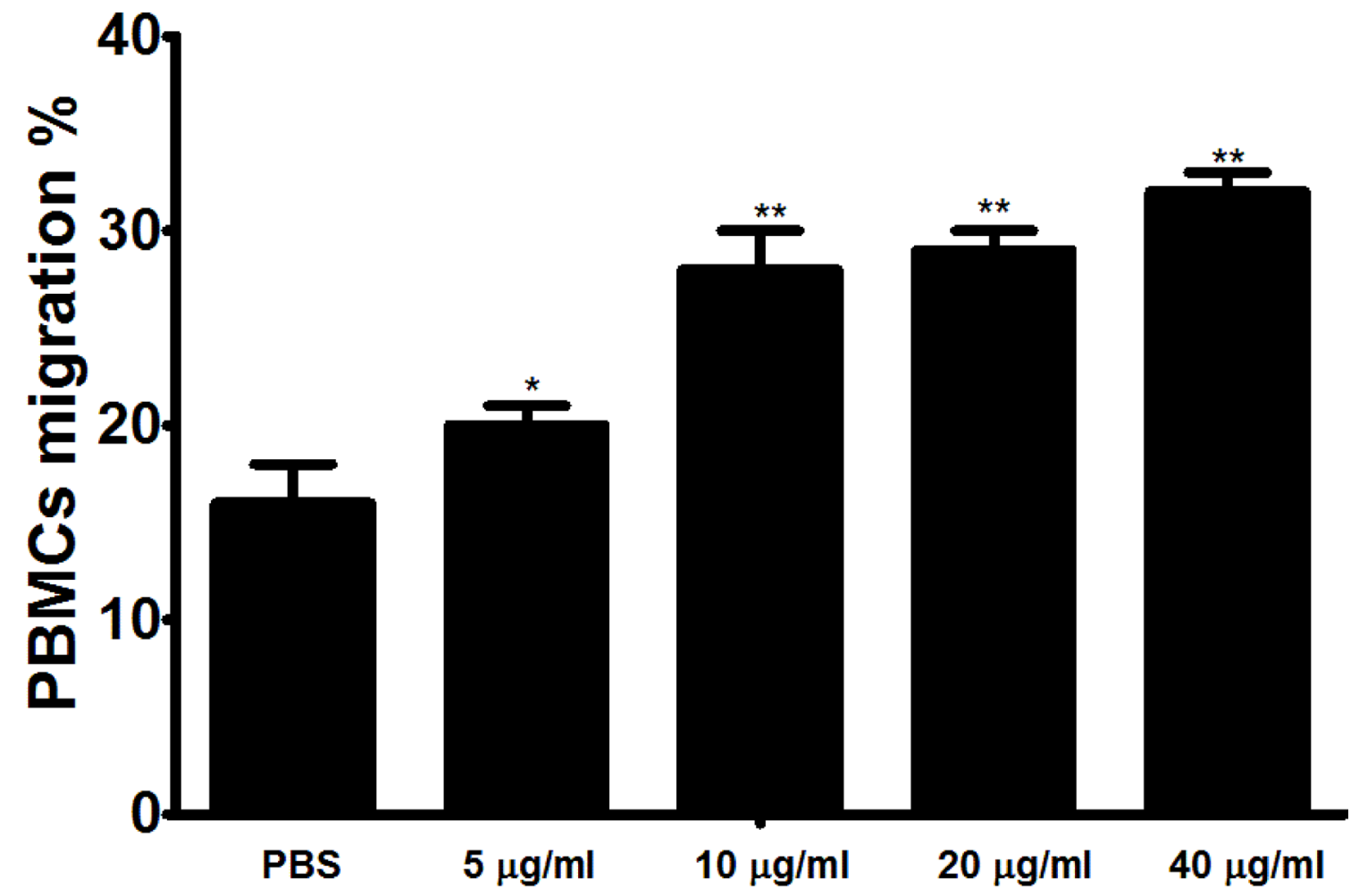

Figure 6: Impact of the various concentration of rHcES-24 on PBMC migration. PBMC were treated with control buffer and different concentrations of rHcES-24. Then the random migration was determined. The difference between the mean values was calculated using ANOVA. Data are representative of 3 independent experiments; ${ }^{*} p<0.01$ and ${ }^{* *} p<0.001$ versus the control. 


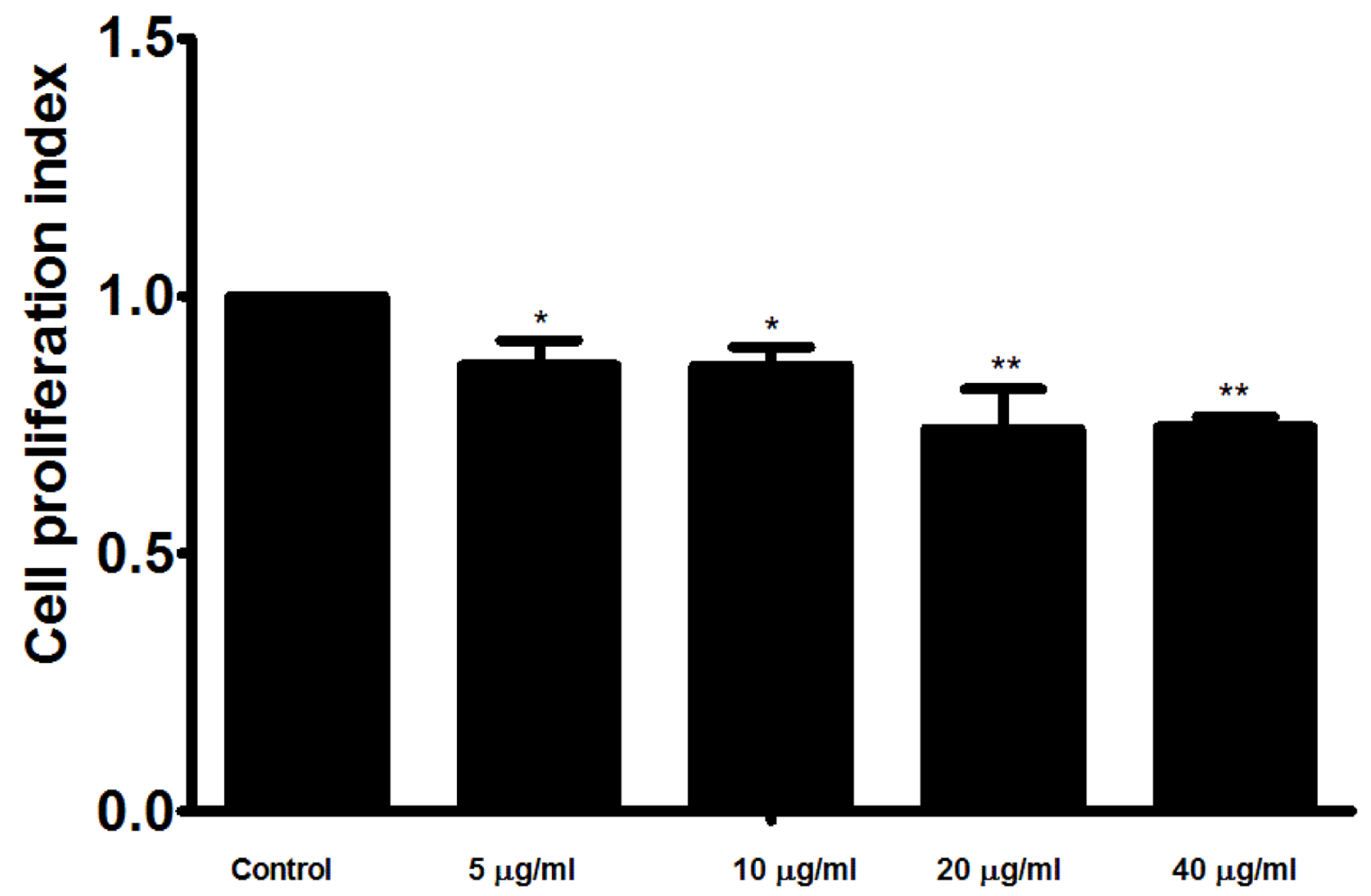

Figure 7: Effects of rHcES-24 on PBMCs proliferation. Cells s were activated with ConA and incubated at the same time with serial concentrations of rHcES-24 at $37^{\circ} \mathrm{C}$ and $5 \% \mathrm{CO}_{2}$. The proliferation was measured by CCK-8 incorporation after $72 \mathrm{~h}$. Cell proliferation index was calculated considering the $\mathrm{OD}_{450}$ values in controls as $100 \%$. The data were representative of three independent experiments $(* p<0.01$ and $* * p<0.001)$.

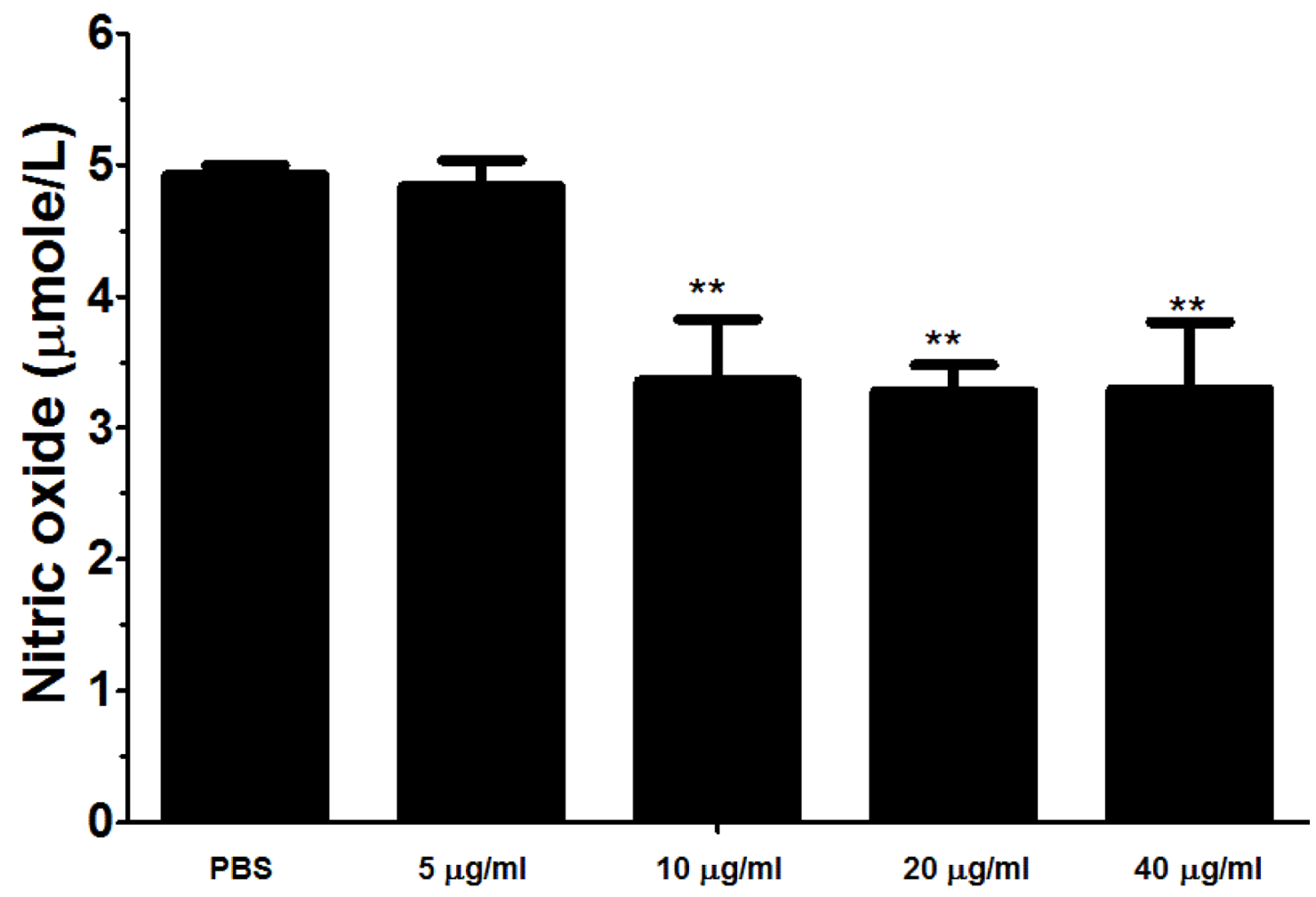

Figure 8: Effects of rHcES-24 on nitric oxide production by PBMCs in vitro. Cells s were activated with ConA and incubated at the same time with serial concentrations of rHcES-24 at $37^{\circ} \mathrm{C}$ and $5 \% \mathrm{CO}_{2}$. The nitrite concentration in the PBMCs was measured by using the Griess assay and used as an indicator of nitric oxide production by the PBMCs. The data were representative of three independent experiments $(* * p<0.001)$. 
of CAP family Hc24 and Hc40 have been recognized from the ES products [14-16]. The role of $H$. contortus CAP protein particularly in immune regulation still not addressed. In the present study we cloned HcES-24 from adult $H$. contortus and rHcES- 24 was used to evaluate the functional role in different regulations of goat PBMCs.

It is generally considered that the main protective immune responses against helminthes including $H$. contortus are Type 2 responses (Th2) associated with secretion of IL-4 and IL-5 [24-26]. In our recent study we found that HcESPs decreased the IL-4 production in vitro [19], Contrary to that, in the present study, we identified that the rHcES-24 could enhance the secretion of IL-4. It indicated that this protein might be initiated the type 2 immune response against the infection of $H$. contortus.

The polarization of Th2 response during helminth infection is usually associated with $\mathrm{T}$ regulatory cells $\left(\mathrm{T}_{\text {Reg }}\right)$. Inducible Treg (iTreg) cells are produced in the periphery and exert their suppressive activity mainly by producing IL-10, which are significantly linked with hyporesponsiveness and susceptibility to infection [27, 28]. IL-10 suppresses the development of allergic Th2 cell responses [29-31]. In our previous study we reported that, HcESPs increased the production of IL-10 in dose dependent manner [19]. Here we found that $\mathrm{rHc}-24$ increased the production of IL-10. Hence, we can say that this protein play some roles in this stimulation of HcESPs on IL-10 production.

IL-17 a cytokine is produced by Th17 cells concerned in the regulation of inflammation [32]. IL-17 is associated with pathogenesis of various parasites including helminths [33-39]. Previously we reported that, HcESPs increased the production of IL-17 [19]. In the present study, we found that $\mathrm{rHc}-24$ increased the production of IL-17; this indicated that $\mathrm{rHc}-24$ could contribute some roles in the HcESPs on IL-17 production.

Cytokine gamma interferon (IFN- $\gamma$ ) secreted mainly from type $1 \mathrm{~T}$ cells (Th1) and NK cells also participates a key role in Th1 and Th2 differentiation [40]. Hsieh et al [41] reported that ESPs of $N$. americanus bind to NK cells and stimulated the Th1 responses by augmentation of IFN- $\gamma$ production. Previously, it was reported that development of type 1 responses produce the resistance to parasite and could inhibit the development of Th2 cells in the immune responses $[42,43]$. Balance between Th1 and $\mathrm{Th} 2$ immune responses could determine the immunity and pathogenesis in parasitic infection [40]. Here, we found that PBMCs incubated with rHcES-24 suppressed the production of IFN- $\gamma$ cytokine level in dose dependent manner. HcESPs suppress the production of IFN- $\gamma$ [19], this protein also. Thus, we can say that this protein play putative role in this suppression of ESP on IFN- $\gamma$ production.

Like other infections, parasites also induce immune cell trafficking to the site of infection, these cell can serve to control the pathogen [44]. Helminths may actively stimulate eosinophil and other lymphocyte migration to the sites of infection, resulting in tissue damage for the worm survival within the host $[45,46]$. Previously reported that GIT nematodes produce a factors that stimulate the cell migration [47]. These finding indicated that helminthes could actively promote cell migration resulting in tissue damage and could provide favorable condition for their survival [46]. In our study rHcES-24 significantly increased the PBMC migration, Our findings fall within the our previous study about the effects of HcESPs on the PBMC migration in vitro [19] which indicated that, this secretory protein also one of the contributor of HcESPs that involved in the PBMCs migration.

An antigen-presenting cell (APC) and T cells can play important role in regulating the proliferation of immune cells and by this means alter the immune response [48]. Loke et al [48] reported that cell proliferative suppression induced by nematode via alternatively activated macrophages by IL-4. Ricci et al [49] demonstrated that hook worm crude antigen decreased the Treg cells and impaired cell proliferation. Previously we found that, HcESPs had a suppressive potential on the cell proliferation [19]. In current study, suppressive activity of the rHcES-24 on PBMC proliferation suggested that, HcES-24 protein is active molecule of HcESPs that impaired the cell proliferation.

Nitric oxide (NO) production considered to be mediating pro as well as anti-inflammatory [50]. NO has been associated with either a decrease in or aggravation of pathogenesis, increase in NO production help pathogen killing by host cell and may also increase the inflammatory responses [51, 52]. In previous studies reported, that NO could played considerable role in immunosuppression by its negative effects on lymphocyte proliferation [53]. [54-56]. Recently we reported that, HcESPs had negative effects on the NO production in vitro [19], therefore the suppression of the NO production by rHcES-24 indicated that HcES-24 might be played some roles in this suppression of HcESPs on NO production.

On the basis of our findings we concluded that HcES-24 is the very important and active protein of HcESPs that played crucial roles in the immune regulations. Our results demonstrated that IL-4, IL-10, IL17 and cell migration were increased by rHcES-24. However, cell proliferation and NO production by goat PBMCs were suppressed by rHcES-24. These findings will not only add to the understanding of the role of HcES24, but might also help to illuminate the mechanisms involved in $H$. contortus immune evasion and host parasite interaction. 


\section{MATERIALS AND METHODS}

\section{Ethics statement}

Animal experiments were conducted following the guidelines of the Animal Ethics Committee, Nanjing Agricultural University, China. All experimental protocols were approved by the Science and Technology Agency of Jiangsu Province. The approval ID is SYXK (SU) 20100005 .

\section{Synthesis of $H$. contortus cDNA}

Total RNA was isolated from Adult worm of $H$. contortus collected from the abomasums of donor goats as described previously [57]. The worms were ground using a pre-chilled mortor and pestle. One $\mathrm{ml}$ of Trizol (Invitrogen) was added and homogenized for 30 minutes. Then $200 \mu 1$ of Tri-chloromethane was added and the mixture was spun at $12,000 \mathrm{rpm}$ for $15 \mathrm{~min}$ at $4^{\circ} \mathrm{C}$. After that, RNA was precipitated from the supernatant by the addition of 0.25 volumes of isopropyl alcohol per each milliliter of Trizol and incubated at $-20^{\circ} \mathrm{C}$ for $30 \mathrm{~min}$. The RNA was pelleted at $12,000 \mathrm{rpm}$ at $4^{\circ} \mathrm{C}$ for $10 \mathrm{~min}$. RNA pellet was washed by $70 \%$ ethanol, dried andresuspended in DEPC-treated water. RNA integrity was checked by agrose gel electrophoresis and quantified by NanoDrop ND-1000 Spectrophotometer. The RNA solution was used in subsequent cDNA preparation immediately. The cDNA was synthesized by reverse transcription reaction using cDNA Kit (TaKaRa Biotechnology) according to the manufacturer's instructions.

\section{Molecular cloning of HcES-24 and expression of recombinant HcES-24 protein (rHcES-24)}

The complete open reading frame (ORF) of HcES24 was amplified by reverse transcription-polymerase chain reaction (RT-PCR) using the designed primers of $H$. contortus $24 \mathrm{kDa}$ excretory/secretory protein mRNA, gene bank accession number AY821551.1.

The sense and antisense primer sequences are as the following: 5'-GAATTCATGTGTCCAGACACCAATG-3' and 5'- AAGCTTTTATGGGGCAATACAGAGA -3' which contained the initiation codon ATG with a EcoRI restriction site at the 5 '-end and the terminus codon TAA with an HindIII restriction site at the 5 (underlined). PCR reaction and amplification were performed using the Thermocycler PCR Machine (Biometra) under following condition: Initial denaturing at $94{ }^{\circ} \mathrm{C}$ for $5 \mathrm{~min}$ (1 cycle), denaturing at $94{ }^{\circ} \mathrm{C}$ for 1 min ( 35 cycles), annealing at $55^{\circ} \mathrm{C}$ for $1.5 \mathrm{~min}(35 \mathrm{cycles})$, extension at $72{ }^{\circ} \mathrm{C}$ for 1.5 $\min \left(35\right.$ cycles) and final extension at $72{ }^{\circ} \mathrm{C}$ for $10 \min (1$ cycle). The PCR products were purified by using E.Z.N.A. Gel Extraction Kit (Omega bio-tech, USA) and ligated into pMD19-T cloning vector (TaKaRa Biotechnology, China) and then transformed into $E$. coli $\mathrm{DH}_{5 \alpha}$ strain. The positive clones were confirmed by double digestion with EcoRI/ HindIII enzymes, and the selected positive recombinant clones were sequenced by Invitrogen Bio-tech (Shanghai, China). The sequence data was assembled and analyzed by DNAssist software version 2.2. The HcES-24 gene was then cloned into EcoRI/ HindIII sites of expression plasmid pET32a $(+)$ vector (Novagen, USA). The recombinant plasmid was sequenced to confirm the correct insertion of HcES-24 gene in the proper reading frame.

The expression of the recombinant fusion protein in $E$. coli BL-2 1 cells (DE3) was induced by isopropy$\beta \mathrm{D}$-thiogala ctoside (IPTG ) at a final concentration of $1 \mathrm{mM}$ for $4 \mathrm{~h}$ at $37^{\circ} \mathrm{C}$ in Luria-Bertini (LB) medium with ampicillin $(100 \mu \mathrm{g} / \mathrm{ml})$. The histidine-tagged fusion protein was purified from the supernatant of bacterial lysates using the His $\bullet$ Bind $^{\circledR}$ Resin Chromatography kit (Novagen) and dialyzed in phosphate buffered saline (PBS, pH 7.4) to remove imidazole. Endotoxins were removed from the recombinant proteins using ToxinEraser ${ }^{\mathrm{TM}}$ Endotoxin Removal kit (GeneScript, USA). The purity and concentration of the purified rHcES-24 was analyzed by $12 \%$ sodium dodecyl sulfate polyacrylamide gelelectrophoresis (SDS- PAGE) followed by Coomassie blue staining.

\section{Sequence alignments and phylogenetic analysis of HcES-24}

Sequence similarity was assessed using proteinprotein basic local alignment search tools BLASTp and BLASTX sequences (http://www.blast.ncbi.nlm.nih. gov/Blast.cgi). HcES-24 sequences were aligned using ClustalX 1.83 program (http://www.clustal.org/). The phylogenetic tree was constructed by aligning the amino acid sequences using the Neighbor-Joining method and plotted and visualized using the Molecular Evolutionary Genetics Analysis 5.1 program (http://www.megasoftware. net/).

\section{Generation of polyclonal antibodies}

To generate polyclonal antibodies against rHcES-24 , $0.4 \mathrm{mg}$ of rHcES-24 was mixed with Freund's complete adjuvant (1:1) and injected subcutaneously into 3 female Sprague Dawley (SD) rats [58, 59]. Rats received four doses of injection with the same proteins at 2 -week intervals. Ten days after the last injection, the rats were anesthetized with diethyl ether, and sera containing specific anti-rHcES-24 antibodies were collected. The 
concentration of antibodies was determined by ELISA. The specific reactivity with $\mathrm{rHcES}-24$ was confirmed by western blot analysis.

\section{Immuno-blot for the rHcES-24}

Purified rHcES-24 were resolved by $10 \%$ SDSPAGE and transferred to polyvinylidene difluoride (PVDF) Membrane (Millipore, USA). Non-specific binding was blocked by incubating the membranes in 5\% skim milk in Tris-buffered saline containing $0.1 \%$ Tween-20 (TBST) for $1 \mathrm{~h}$ at room temperature. The membranes were then washed 5 times ( 5 min each) with TBST, followed by incubation with the primary antibodies (anti-rHcES-24) for $1 \mathrm{~h}$ at $37^{\circ} \mathrm{C}(1: 100$ dilution in TBST). After washing 5 times with TBST, the membranes were incubated with HRP-conjugated rabbit anti-rat IgG (Sigma, USA) for 1 $\mathrm{h}$ at $37{ }^{\circ} \mathrm{C}$ (diluted 1:2000 in TBST). Finally, the bound antibody was detected using 3, 3-diaminobenzidine tetra hydrochloride (DAB) kit (Boster Bio-technology) according to manufacturer's instructions.

\section{Binding of rHcES-24 to goat PBMC}

Freshly isolated PBMCs were incubated in the presence and absence (control) of rHcES-24 $(5 \mu \mathrm{g} / \mathrm{ml})$ for 1 $\mathrm{h}$ at $37^{\circ} \mathrm{C}$. Confirmation of binding was determined by an immunofluorescence assay (IFA) as described by Yuan et al. [54]. Briefly, washed cells $\left(10^{5} / \mathrm{ml}\right)$ were fixed with $4 \%$ paraformaldehyde on a poly-L-lysine-coated glass slide. The cells were then treated with blocking solution (4\% BSA in PBS) for 30 min to minimize background staining. After sequential incubation with rat anti-rHcES-24 IgG $(1: 100)$ for $2 \mathrm{~h}$ and a secondary antibody (1:300) coupled to the fluorescent dye Cy3 (Beyotime, Jiangsu, China) for $1 \mathrm{~h}$, nuclear staining with 2-(4-amidinophenyl)-6-indole carbamidinedihydrochloride (DAPI, $1.5 \mu \mathrm{M}$; Sigma, MO, USA) was performed for $6 \mathrm{~min}$. Then, protein localization was determined by observing the staining patterns with a $100 \times$ oil objective lens on a laser scanning confocal microscope (L SM710, Zeiss, Jena, Germany). Digital images were captured using the Zeiss microscope software package ZEN 2012 (Zeiss, Jena, Germany).

\section{Detection of the cytokine levels by ELISA of PBMCs treated with rHcES-24}

The freshly isolated PBMCs were re-suspended to a final density of $5 \times 10^{6} / \mathrm{ml}$ in complete medium (RPMI 1640 supplemented with $100 \mathrm{U} / \mathrm{ml}$ penicillin, $100 \mu \mathrm{g} /$ $\mathrm{ml}$ streptomycin, $2 \mathrm{mM}$ L-glutamine, 10\% FCS). In the test groups, cells were treated with ConA $(10 \mu \mathrm{g} / \mathrm{ml})$ and different concentration of the rHcES-24 $(5,10,20$, and $40 \mu \mathrm{g} / \mathrm{ml})$. The control groups were treated with ConA in equal volume of PBS or ConA and recombinant protein of empty pET32a. Then, the cells were seeded into 24well plates $(1 \mathrm{ml} /$ well) and cultured for $24 \mathrm{~h}$ in $5 \% \mathrm{CO} 2$ atmosphere at $37^{\circ} \mathrm{C}$. The plates were then centrifuged at $200 \times \mathrm{g}$ for $15 \mathrm{~min}$ and the supernatants were collected. The levels of IL-4, IL-10, IL-17 and IFN- $\gamma$ in supernatants were determined using commercially available goat ELISA kits (Jian cheng Biotech, China). The cell viability was assessed by means of the trypan blue exclusion test before the incubation of PBMCs with rHcES-24. Three individual experiments were performed.

\section{Cell migration assay}

The cell migration assay was performed using a Transwell system (Corning, USA), this allowed cells to migrate throughout an $8 \mu \mathrm{m}$ pore size polycarbonate membrane [54]. The treatment group was incubated with different concentrations of rHcES-24 (5, 10, 20, and $40 \mu \mathrm{g} / \mathrm{ml}$ ) and the control group was treated with an equal volume of PBS. Cells that migrated through the membrane into the lower chamber were calculated with a Neubauer counting chamber and the results were presented as percentages of the seeded PBMC. Each experiment was performed in triplicate.

\section{Cell proliferation assay}

Cell proliferation assay was performed as previously described [60]. Briefly, $100 \mu \mathrm{l}$ of cell suspension $\left(1 \times 10^{6}\right.$ cells $/ \mathrm{ml})$ were activated with ConA $(10 \mu \mathrm{g} / \mathrm{ml})$ and a serial concentrations of rHcES-24 $(5,10,20$, and $40 \mu \mathrm{g} /$ $\mathrm{ml})$. The control group was treated with ConA in equal volume of PBS. The plate was cultured at $37^{\circ} \mathrm{C}$ and $5 \%$ $\mathrm{CO}_{2}$ for $72 \mathrm{~h}$. Then $10 \mu \mathrm{l}$ of CCK-8 solutions (Beyotime Biotechnology, China) were added to each well of the plates $4 \mathrm{~h}$ before harvesting and the absorbance values at $450 \mathrm{~nm}\left(\mathrm{OD}_{450}\right)$ were measured using a microplate reader (Thermo Scientific, USA). The $\mathrm{OD}_{450}$ of controls were set as $100 \%$. Cell proliferation index was calculated by the formula: $\mathrm{OD}_{450} \mathrm{rHcES}-24 / \mathrm{OD}_{450}$ control. Each experiment was performed in triplicate

\section{Nitric oxide production assay}

The goat PBMCs were harvested and washed twice with PBS. Then, $100 \mu \mathrm{l}$ of cells $\left(1 \times 10^{6}\right.$ cells $\left./ \mathrm{ml}\right)$ were incubated either with PBS and a serial concentrations of rHcES-24 $(5,10,20$, and $40 \mu \mathrm{g} / \mathrm{ml})$ in 96-well plates in DMEM medium. Production of nitric oxide by PBMCs was determine $\mathrm{d}$ by measurement of intracellular nitrite in the PBMC by using the Griess assay [61] according to the instruction of Total Nitric Oxide Assay Kit (Beyotime Biotechnology, China). Absorbance of the colored solution 
at $540 \mathrm{~nm}$ (OD540) in each well was measured using a plate reader (Bio-Rad Laboratories, USA). Absorbance values were converted to micromoles per liter $(\mu \mathrm{mol} / \mathrm{L})$ using a standard curve that was generated by addition of 0 to $80 \mu \mathrm{mol} / \mathrm{L}$ sodium nitrite to fresh culture media. Three individual experiments were performed.

\section{FUNDING}

The project supports were provided by the "National Key Basic Research Program (973 program) of China" (Grant No. 2015CB150300) and by the Priority Academic Program Development of Jiangsu Higher Education Institutions (PAPD).

\section{CONFLICTS OF INTERESTS}

The authors declare that they have no competing interests.

\section{REFERENCES}

1. Nikolaou S and Gasser RB. Prospects for exploring molecular developmental processes in Haemonchus contortus. Int J Parasitol. 2006; 36(8):859-868.

2. Blackburn HD, Rocha JL, Figueiredo EP, Berne ME, Vieira LS, Cavalcante AR and Rosa JS. Interaction of parasitism and nutrition in goats: effects on haematological parameters, correlations, and other statistical associations. Vet Parasitol. 1992; 44(3-4):183-197.

3. Jasmer DP, Lahmers KK and Brown WC. Haemonchus contortus intestine: a prominent source of mucosal antigens. Parasite immunology. 2007; 29(3):139-151.

4. Mohandas N, Young ND, Jabbar A, Korhonen PK, Koehler AV, Amani P, Hall RS, Sternberg PW, Jex AR, Hofmann $A$ and Gasser RB. The barber's pole worm CAP protein superfamily - A basis for fundamental discovery and biotechnology advances. Biotechnology Advances. 2015; 33(8):1744-1754.

5. Schwarz EM, Korhonen PK, Campbell BE, Young ND, Jex AR, Jabbar A, Hall RS, Mondal A, Howe AC, Pell J, Hofmann A, Boag PR, Zhu X-Q, Gregory TR, Loukas A, Williams BA, et al. The genome and developmental transcriptome of the strongylid nematode Haemonchus contortus. Genome Biology. 2013; 14(8):R89-R89.

6. Laing R, Kikuchi T, Martinelli A, Tsai IJ, Beech RN, Redman E, Holroyd N, Bartley DJ, Beasley H, Britton C, Curran D, Devaney E, Gilabert A, Hunt M, Jackson F, Johnston SL, et al. The genome and transcriptome of Haemonchus contortus, a key model parasite for drug and vaccine discovery. Genome Biology. 2013; 14(8):R88-R88.

7. Gibbs GM, Roelants $\mathrm{K}$ and O'Bryan MK. The CAP superfamily: cysteine-rich secretory proteins, antigen 5, and pathogenesis-related 1 proteins - roles in reproduction, cancer, and immune defense. Endocrine reviews. 2008;
29(7):865-897.

8. Asojo OA. Structure of a two-CAP-domain protein from the human hookworm parasite Necator americanus. Acta Crystallographica Section D: Biological Crystallography. 2011; 67(Pt 5):455-462.

9. Cantacessi C, Campbell BE, Visser A, Geldhof P, Nolan MJ, Nisbet AJ, Matthews JB, Loukas A, Hofmann A, Otranto D, Sternberg PW and Gasser RB. A portrait of the "SCP/TAPS" proteins of eukaryotes - developing a framework for fundamental research and biotechnological outcomes. Biotechnol Adv. 2009; 27(4):376-388.

10. Zhan B, Liu Y, Badamchian M, Williamson A, Feng J, Loukas A, Hawdon JM and Hotez PJ. Molecular characterisation of the Ancylostoma-secreted protein family from the adult stage of Ancylostoma caninum. International journal for parasitology. 2003; 33(9):897-907.

11. van Loon LC, Rep M and Pieterse CM. Significance of inducible defense-related proteins in infected plants. Annual review of phytopathology. 2006; 44:135-162.

12. Hawdon JM, Jones BF, Hoffman DR and Hotez PJ. Cloning and characterization of Ancylostoma-secreted protein. A novel protein associated with the transition to parasitism by infective hookworm larvae. The Journal of biological chemistry. 1996; 271(12):6672-6678.

13. Moser JM, Freitas T, Arasu P and Gibson G. Gene expression profiles associated with the transition to parasitism in Ancylostoma caninum larvae. Mol Biochem Parasitol. 2005; 143(1):39-48.

14. Rehman A and P. Jasmer D. A tissue specific approach for analysis of membrane and secreted protein antigens from Haemonchus contortus gut and its application to diverse nematode species. Molecular and Biochemical Parasitology. 1998; 97(1-2):55-68.

15. Schallig HD, van Leeuwen MA, Verstrepen BE and Cornelissen AW. Molecular characterization and expression of two putative protective excretory secretory proteins of Haemonchus contortus. Molecular and biochemical parasitology. 1997; 88(1-2):203-213.

16. Yatsuda AP, Krijgsveld J, Cornelissen AW, Heck AJ and de Vries E. Comprehensive analysis of the secreted proteins of the parasite Haemonchus contortus reveals extensive sequence variation and differential immune recognition. The Journal of biological chemistry. 2003; 278(19):1694116951.

17. Takats C, Schallig HD, Van Leeuwen MA and Hendrikx WM. Immune responses of sheep to microdissected parts of Haemonchus contortus. International journal for parasitology. 1995; 25(7):857-860.

18. Schallig HD, van Leeuwen MA and Cornelissen AW. Protective immunity induced by vaccination with two Haemonchus contortus excretory secretory proteins in sheep. Parasite immunology. 1997; 19(10):447-453.

19. Gadahi JA, Yongqian B, Ehsan M, Zhang ZC, Wang $\mathrm{S}$, Yan RF, Song XK, Xu LX and Li XR. Haemonchus 
contortus excretory and secretory proteins (HcESPs) suppress functions of goat PBMCs in vitro. Oncotarget. 2016;7:35670-35679. doi: 10.18632/oncotarget.9589.

20. Gadahi JA, Wang S, Bo G, Ehsan M, Yan R, Song X, $\mathrm{Xu} \mathrm{L}$ and Li X. Proteomic Analysis of the Excretory and Secretory Proteins of Haemonchus contortus (HcESP) Binding to Goat PBMCs In Vivo Revealed Stage-Specific Binding Profiles. PLoS ONE. 2016; 11(7):e0159796.

21. Datu BJ, Gasser RB, Nagaraj SH, Ong EK, O’Donoghue P, McInnes R, Ranganathan S and Loukas A. Transcriptional changes in the hookworm, Ancylostoma caninum, during the transition from a free-living to a parasitic larva. PLoS neglected tropical diseases. 2008; 2(1):e130.

22. Asojo OA, Goud G, Dhar K, Loukas A, Zhan B, Deumic V, Liu S, Borgstahl GE and Hotez PJ. X-ray structure of NaASP-2, a pathogenesis-related-1 protein from the nematode parasite, Necator americanus, and a vaccine antigen for human hookworm infection. Journal of molecular biology. 2005; 346(3):801-814.

23. Asojo O. Structure of a two-CAP-domain protein from the human hookworm parasite Necator americanus. Acta Crystallographica Section D. 2011; 67(5):455-462.

24. de Rezende MC, Araujo ES, Moreira JM, Rodrigues VF, Rodrigues JL, Pereira CA and Negrao-Correa D. Effect of different stages of Schistosoma mansoni infection on the parasite burden and immune response to Strongyloides venezuelensis in co-infected mice. Parasitology research. 2015; 114(12):4601-4616.

25. Andreasen A, Petersen HH, Kringel H, Iburg TM, Skovgaard K, Dawson H, Urban JF, Jr. and Thamsborg SM. Immune and inflammatory responses in pigs infected with Trichuris suis and Oesophagostomum dentatum. Vet Parasitol. 2015; 207(3-4):249-258.

26. Shakya KP, Miller JE and Horohov DW. A Th2 type of immune response is associated with increased resistance to Haemonchus contortus in naturally infected Gulf Coast Native lambs. Vet Parasitol. 2009; 163(1-2):57-66.

27. McSorley HJ and Maizels RM. Helminth infections and host immune regulation. Clinical microbiology reviews. 2012; 25(4):585-608.

28. Adalid-Peralta L, Fragoso G, Fleury A and Sciutto E. Mechanisms underlying the induction of regulatory $T$ cells and its relevance in the adaptive immune response in parasitic infections. International journal of biological sciences. 2011; 7(9):1412-1426.

29. Levings MK, Sangregorio R, Galbiati F, Squadrone S, de Waal Malefyt R and Roncarolo MG. IFN-alpha and IL-10 induce the differentiation of human type $1 \mathrm{~T}$ regulatory cells. Journal of immunology. 2001; 166(9):5530-5539.

30. Taylor A, Verhagen J, Blaser K, Akdis M and Akdis CA. Mechanisms of immune suppression by interleukin-10 and transforming growth factor- $\beta$ : the role of T regulatory cells. Immunology. 2006; 117(4):433-442.

31. Grencis RK, Humphreys NE and Bancroft AJ. Immunity to gastrointestinal nematodes: mechanisms and myths. Immunological Reviews. 2014; 260(1):183-205.

32. Ferretti S, Bonneau O, Dubois GR, Jones CE and Trifilieff A. IL-17, produced by lymphocytes and neutrophils, is necessary for lipopolysaccharide-induced airway neutrophilia: IL-15 as a possible trigger. Journal of immunology. 2003; 170(4):2106-2112.

33. da Matta Guedes PM, Gutierrez FR, Maia FL, Milanezi CM, Silva GK, Pavanelli WR and Silva JS. IL-17 produced during Trypanosoma cruzi infection plays a central role in regulating parasite-induced myocarditis. PLoS neglected tropical diseases. 2010; 4(2):e604.

34. Guiton R, Vasseur V, Charron S, Arias MT, Van Langendonck N, Buzoni-Gatel D, Ryffel B and DimierPoisson I. Interleukin 17 receptor signaling is deleterious during Toxoplasma gondii infection in susceptible BL6 mice. The Journal of infectious diseases. 2010; 202(3):427435 .

35. Fu Y, Wang W, Tong J, Pan Q, Long Y, Qian W and Hou X. Th17: a new participant in gut dysfunction in mice infected with Trichinella spiralis. Mediators of inflammation. 2009; 2009:517052.

36. Larkin B, Smith P, Ponichtera H, Shainheit M, Rutitzky L and Stadecker M. Induction and regulation of pathogenic Th17 cell responses in schistosomiasis. Seminars in Immunopathology. 2012; 34(6):873-888.

37. Katawa G, Layland LE, Debrah AY, von Horn C, Batsa L, Kwarteng A, Arriens S, D WT, Specht S, Hoerauf A and Adjobimey T. Hyperreactive onchocerciasis is characterized by a combination of Th17-Th2 immune responses and reduced regulatory $\mathrm{T}$ cells. PLoS neglected tropical diseases. 2015; 9(1):e3414.

38. Mbow M, Larkin BM, Meurs L, Wammes LJ, de Jong SE, Labuda LA, Camara M, Smits HH, Polman K, Dieye TN, Mboup S, Stadecker MJ and Yazdanbakhsh M. T-helper 17 cells are associated with pathology in human schistosomiasis. The Journal of infectious diseases. 2013; 207(1):186-195.

39. Rutitzky LI and Stadecker MJ. CD4 T cells producing pro-inflammatory interleukin-17 mediate high pathology in schistosomiasis. Memorias do Instituto Oswaldo Cruz. 2006; 101 Suppl 1:327-330.

40. Uchikawa R, Matsuda S and Arizono N. Suppression of Gamma Interferon Transcription and Production by Nematode Excretory-Secretory Antigen during Polyclonal Stimulation of Rat Lymph Node T Cells. Infection and Immunity. 2000; 68(11):6233-6239.

41. Hsieh GC-F, Loukas A, Wahl AM, Bhatia M, Wang Y, Williamson AL, Kehn KW, Maruyama H, Hotez PJ, Leitenberg D, Bethony $\mathrm{J}$ and Constant SL. A Secreted Protein from the Human Hookworm Necator americanus Binds Selectively to NK Cells and Induces IFN- $\gamma$ Production. The Journal of Immunology. 2004; 173(4):2699-2704. 
42. Coomes SM, Pelly VS, Kannan Y, Okoye IS, Czieso S, Entwistle LJ, Perez-Lloret J, Nikolov N, Potocnik AJ, Biró $\mathrm{J}$, Langhorne $\mathrm{J}$ and Wilson MS. IFN $\gamma$ and IL-12 Restrict Th2 Responses during Helminth/Plasmodium Co-Infection and Promote IFN $\gamma$ from Th2 Cells. PLoS Pathogens. 2015; 11(7):e1004994.

43. Duerr CU, McCarthy CDA, Mindt BC, Rubio M, Meli AP, Pothlichet J, Eva MM, Gauchat J-F, Qureshi ST, Mazer BD, Mossman KL, Malo D, Gamero AM, Vidal SM, King IL, Sarfati M, et al. Type I interferon restricts type 2 immunopathology through the regulation of group 2 innate lymphoid cells. Nat Immunol. 2016; 17(1):65-75.

44. McGovern KE and Wilson EH. Role of Chemokines and Trafficking of Immune Cells in Parasitic Infections. Current immunology reviews. 2013; 9(3):157-168.

45. Wang W, Yuan C, Wang S, Song X, Xu L, Yan R, Hasson IA and Li X. Transcriptional and proteomic analysis reveal recombinant galectins of Haemonchus contortus downregulated functions of goat $\mathrm{PBMC}$ and modulation of several signaling cascades in vitro. Journal of proteomics. 2014; 98(0):123-137.

46. Turner DG, Wildblood LA, Inglis NF and Jones DG. Characterization of a galectin-like activity from the parasitic nematode, Haemonchus contortus, which modulates ovine eosinophil migration in vitro. Veterinary immunology and immunopathology. 2008; 122(1-2):138-145.

47. Wildblood LA, Kerr K, Clark DA, Cameron A, Turner DG and Jones DG. Production of eosinophil chemoattractant activity by ovine gastrointestinal nematodes. Veterinary immunology and immunopathology. 2005; 107(1-2):57-65.

48. Loke P, MacDonald AS, Robb A, Maizels RM and Allen JE. Alternatively activated macrophages induced by nematode infection inhibit proliferation via cell-to-cell contact. European journal of immunology. 2000; 30(9):2669-2678.

49. Ricci ND, Fiúza JA, Bueno LL, Cançado GGL, GazzinelliGuimarães PH, Martins VG, Matoso LF, de Miranda RRC, Geiger SM, Correa-Oliveira R, Gazzinelli A, Bartholomeu DC and Fujiwara RT. Induction of CD4(+)CD25(+) FOXP3(+) Regulatory T Cells during Human Hookworm Infection Modulates Antigen-Mediated Lymphocyte Proliferation. PLoS neglected tropical diseases. 2011; 5(11):e1383.

50. Bogdan C. Nitric oxide and the immune response. Nat Immunol. 2001; 2(10):907-916.

51. Bian K, Zhong M, Harari Y, Lai M, Weisbrodt N and Murad F. Helminth regulation of host IL-4Ralpha/Stat6 signaling: mechanism underlying NOS-2 inhibition by Trichinella spiralis. Proceedings of the National Academy of Sciences of the United States of America. 2005; 102(11):3936-3941.
52. Brunet LR. Nitric oxide in parasitic infections. International immunopharmacology. 2001; 1(8):1457-1467.

53. Dondji B, Bungiro RD, Harrison LM, Vermeire JJ, Bifulco C, McMahon-Pratt D and Cappello M. Role for Nitric Oxide in Hookworm-Associated Immune Suppression. Infection and Immunity. 2008; 76(6):2560-2567.

54. Yuan C, Zhang H, Wang W, Li Y, Yan R, Xu L, Song X and Li X. Transmembrane protein $63 \mathrm{~A}$ is a partner protein of Haemonchus contortus galectin in the regulation of goat peripheral blood mononuclear cells. Parasites \& vectors. 2015; 8(1):211.

55. Bian M, Li S, Wang X, Xu Y, Chen W, Zhou C, Chen X, He L, Xu J, Liang C, Wu Z, Huang Y, Li X and Yu X. Identification, immunolocalization, and immunological characterization of nitric oxide synthase-interacting protein from Clonorchis sinensis. Parasitology research. 2014; 113(5):1749-1757.

56. Nahrevanian H. Involvement of nitric oxide and its up/ down stream molecules in the immunity against parasitic infections. The Brazilian journal of infectious diseases. 2009; 13(6):440-448.

57. Sun Y, Yan R, Muleke CI, Zhao G, Xu 1 and Li X. Recombinant Galectins of Haemonchus contortus Parasite Induces Apoptosis in the Peripheral Blood Lymphocytes of Goat. International Journal of Peptide Research and Therapeutics. 2006; 13(3):387-392.

58. Han K, Xu L, Yan R, Song X and Li X. Molecular cloning, expression and characterization of enolase from adult Haemonchus contortus. Research in veterinary science. 2012; 92(2):259-265.

59. Wang W, Yuan C, Wang S, Song X, Xu L, Yan R, Hasson IA and Li X. Transcriptional and proteomic analysis reveal recombinant galectins of Haemonchus contortus downregulated functions of goat $\mathrm{PBMC}$ and modulation of several signaling cascades in vitro. J Proteomics. 2014; 98(0):123-137.

60. Wang W, Wang S, Zhang H, Yuan C, Yan R, Song X, Xu L and Li X. Galectin Hco-gal-m from Haemonchus contortus modulates goat monocytes and $\mathrm{T}$ cell function in different patterns. Parasites \& vectors. 2014; 7:342.

61. Green LC, Wagner DA, Glogowski J, Skipper PL, Wishnok JS and Tannenbaum SR. Analysis of nitrate, nitrite, and $[15 \mathrm{~N}]$ nitrate in biological fluids. Analytical biochemistry. 1982; 126(1):131-138. 